\title{
Prologue: The "Ready-to-Go" Lymphocytes of the Immune System
}

As outlined in Part III, Sects. 8.4 and 8.5, the whole family of mammalian innate immune cells includes ILCs and unconventional T cells with partial innate function. So far, three groups of ILCs, that is, ILC1, ILC2, and ILC3, have been classified and categorized based upon shared expression of surface markers, transcription factors, and effector cytokines. Innate lymphoid cells, compared to typical lymphoid cells, are characterized by three main features: (1) the absence of RAG-dependent rearrangement of antigen receptors, (2) a lack of phenotypical markers of myeloid cells and DCs, and (3) the particular lymphoid morphology. Unconventional T cells refer to lymphocytes with partial innate function and, thus, operate in the afferent phase of the immune response. These cells are characterized by semi-invariant, invariant, or even germline-encoded TCRs and include NKT cells, $\gamma \delta$ T cells, and MAIT cells. These cells have formerly been described as innate lymphocytes; sometimes they are also called "innate-like" $\mathrm{T}$ lymphocytes because they have several abilities typical of cells of the innate immune system. The characteristic feature of some of these TCR-bearing T cell subsets assigning them to the family of innate immune cells is their equipment with certain NK cell markers. In Part VII of this book, some of the effector responses of these two critical families of innate immune cells are depicted by highlighting mechanisms of their activation including the action of MAMPs and/ or DAMPs. 